\title{
Metallothionein expression in slow- vs. fast-twitch muscle fibers following 4 weeks of streptozotocin-induced type 1 diabetes
}

\author{
Pamela Mondragon $^{\mathrm{a}}$ and Andreas Bergdahl ${ }^{\mathrm{a} *}$ \\ a Department of Exercise Science, Concordia University, 7141 Sherbrooke West, Montreal, QC H4B 1R6, \\ Canada \\ *andreas.bergdahl@concordia.ca
}

\section{OPEN ACCESS}

\section{Citation: Mondragon P and Bergdahl A. 2018. Metallothionein expression in slow- vs. fast-twitch muscle fibers following 4 weeks of streptozotocin-induced type I diabetes. FACETS 3: 315-325. doi: 10.1 139 / facets-2017-0058 \\ Handling Editor: Charles Couillard \\ Received: May 29, 2017}

Accepted: December 18, 2017

Published: March 22, 2018

Copyright: (C) 2018 Mondragon and Bergdahl. This work is licensed under a Creative Commons Attribution 4.0 International License (CC BY 4.0), which permits unrestricted use, distribution, and reproduction in any medium, provided the original author(s) and source are credited.

Published by: Canadian Science Publishing

\begin{abstract}
Type 1 diabetes (T1DM) is known to cause an increase in reactive oxygen species (ROS) and elevated intracellular glucose levels. We investigated the metallothionein I and II (MT I+II) antioxidants expression in soleus (mainly slow-twitch) and plantaris (predominantly fast-twitch) skeletal muscle using a rodent model of streptozotocin-induced diabetes. The presence of oxidative stress was confirmed by the detection of increased levels of protein carbonyl formation in the diabetic tissues. $\mathrm{DAB}\left(3,3^{\prime}\right.$-diaminobenzidine) immunostaining and Western blotting analyses demonstrated that MT I+II expression was significantly upregulated in the diabetic soleus and plantaris muscle tissues compared with their respective controls. Moreover, no significant difference was detected between the plantaris and soleus controls or between the plantaris and soleus diabetic tissues. These findings suggest that there is an increase in MT protein expression in the soleus and plantaris muscles associated with the induction of T1DM. A better understanding of the molecular mechanisms that allow MT to prevent the oxidative stress associated with diabetes could lead to a novel therapeutic strategy for this chronic disease and its associated complications.
\end{abstract}

Key words: metallothionein, type 1 diabetes, hyperglycemia, streptozotocin, skeletal muscle, oxidative stress, reactive oxygen species

\section{Introduction}

Diabetes mellitus is a major public health concern with an increasing prevalence worldwide over the last decades (World Health Organization 2016). Type 1 diabetes mellitus (T1DM) is a disease characterized by the destruction of the pancreatic $\beta$ cells by immune $\mathrm{T}$ cells, which results in the inability to secrete insulin, ensuing high blood glucose levels (i.e., hyperglycemia) (Van Belle et al. 2011). A widely used model to study T1DM in rodents is the injection of streptozotocin (STZ), whose methylnitrosourea moiety triggers the specific destruction of pancreatic $\beta$ cells via DNA alkylation (Wu and Yan 2015).

Oxidative stress occurs when there is an excess production of reactive oxygen species (ROS) and (or) an insufficient removal of them by the antioxidant defense system (Maritim et al. 2003). Moreover, it has been suggested that oxidative stress contributes to the development of T1DM by directly inducing $\beta$-cell damage and by facilitating the autoimmune functions of T cells (Liu et al. 2016). Once diabetes is established, hyperglycemia further induces an increased production of ROS thereby contributing to the development of diabetic complications (King and Loeken 2004; Giacco and Brownlee 2010). 
In diabetic skeletal muscle, oxidative stress contributes to the onset of insulin resistance and to impaired muscle growth and repair (Coleman et al. 2015). Moreover, muscles composed mainly of fast-twitch fibers (i.e., highly oxidative capacity) exhibit an increased loss of function and a more severe atrophy than muscles composed predominantly of slow-twitch muscle fibers (i.e., low oxidative capacity) (Krause et al. 2011). Therefore, the skeletal muscle of STZ-rodents seems to be affected by oxidative stress in a fiber-type-dependent manner.

Metallothioneins (MT) are intracellular low molecular weight, cysteine-rich antioxidant proteins that are expressed in response to oxidative stress as well as play a role in zinc homeostasis and heavy metal detoxification (Carpenè et al. 2007). It has been reported that zinc-induced MT overexpression prevents partially the development of diabetes in STZ-induced rats and that this effect coincides with a decrease of lipid peroxidation (Yang and Cherian 1994). Managing diabetes properly can contribute to preventing the serious complications associated with this disease including cardiovascular disease and damage to the kidneys, the nerves, and the retina (Asmat et al. 2016). A previous study has shown that MT induction contributes to prevent cardiomyopathy in diabetic mice by inhibiting the nitrative damage caused by peroxynitrite and NAPDH (NOX) activation (Cai 2006). Moreover, it has been suggested that zinc-induced MT upregulation protects against diabetes-associated hepatic damage by inhibiting the expression of endoplasmic reticulum (ER) stress factors and two ER-associated cell death pathways (Liang et al. 2015). Taken together, these studies demonstrate that MT may protect against the development of diabetes and its associated complications.

Antioxidants such as superoxide dismutase, glutathione peroxidase, and catalase have been reported to exhibit a higher protein expression in fast-twitch fibers than in slow-twitch fibers (Powers et al. 2011), which suggests that MT could likely have a fiber-type dependent protein expression pattern too. The aim of the present study was to investigate the effects of STZ-induced T1DM on the MT I+II expression in the soleus and plantaris muscles. Given the presence of oxidative stress in diabetes and the different muscle fiber types of skeletal muscle, we hypothesize there to be a difference in the MT expression in the soleus and plantaris muscles of diabetic and nondiabetic rats.

\section{Materials and methods}

\section{Animal care and induction of diabetes by STZ}

Male Wistar rats weighting approximately 200-250 g (Charles River Breeding Farms, St. Constant, Quebec, Canada) were randomly assigned to either the control $(n=8)$ or the STZ-induced diabetic group $(n=8)$. The animals were individually stored in a $22^{\circ} \mathrm{C}$ environment, on a $12: 12 \mathrm{~h}$ light:dark cycle and were given a standard dry chow diet and water ad libitum. All experimental procedures were carried out in accordance with the guidelines of the Canadian Council in Animal Care and the protocols were approved by the Animal Ethics Committee of Concordia University (animal ethics certificate number BERG 2010).

The induction of diabetes was done according to the protocol used by Larsen et al. (2015). Briefly, the experimental group was given a single bolus injection of STZ into the tail vein at a dose of $65 \mathrm{mg} / \mathrm{kg}$ (Sigma, St. Louis, Missouri, USA). The control rats received a citrate buffer injection. An Adventurer balance (OHAUS Corporation, Parsippany, New Jersey, USA) and a Precision Xtra glucose meter (Abbot Laboratories, Mississauga, Ontario, Canada) were used to monitor the weight and the blood glucose concentrations every second day during the 4 weeks post-STZ injection. The parameters used to confirm the presence of diabetes in the experimental group were an increase in blood glucose concentration and a reduction in weight gain. The rodents were euthanized by $\mathrm{CO}_{2}$ inhalation. Soleus and plantaris muscle tissues were isolated, snap frozen in liquid nitrogen, and then kept at $-80^{\circ} \mathrm{C}$ for further MT expression analysis. 


\section{Tissue processing}

The frozen soleus and plantaris skeletal muscle tissues were sectioned using a Leica CM3050 S cryostat (set at $-20^{\circ} \mathrm{C}$ ). Optimal cutting temperature embedding medium was used to orient the tissue under the microscope and give it support. The muscle tissues were cut into $12 \mu \mathrm{m}$ sections. These sections were immediately transferred onto glass slides and stored in the $-20{ }^{\circ} \mathrm{C}$ freezer for later use in 3,3'-diaminobenzidine (DAB) immunostaining.

\section{DAB immunostaining}

For this method, four rats were examined for each diabetic and control group. First, the sections were fixed by $4 \%$ paraformaldehyde for $10 \mathrm{~min}$ at room temperature. The sections were then washed in phosphate-buffered saline (PBS) for $10 \mathrm{~min}$ followed by incubation in $0.3 \% \mathrm{H}_{2} \mathrm{O}_{2}$ in PBS for 5 min to block endogenous peroxidase activity. The sections were washed in PBS for 5 min before they were incubated in $5 \%$ bovine serum albumin (BSA) blocking buffer for $60 \mathrm{~min}$ at room temperature in a humidified chamber. The BSA blocking buffer was subsequently drained from the sections by using PBS as a wash buffer. Then the sections were incubated overnight at $4{ }^{\circ} \mathrm{C}$ with the primary MT-I+II antibody (ab12228; Abcam, Toronto, Ontario, Canada) diluted in PBS with 1\% BSA 1:500 followed by horseradish peroxidase (HRP)-conjugated rabbit anti-mouse immunoglobulin G (IgG) (ab6728; Abcam) diluted in PBS with 1\% BSA 1:1000 in a dark humidified chamber at room temperature for $35 \mathrm{~min}$. The immunostaining was visualized by applying $100 \mu \mathrm{L}$ of freshly made DAB peroxidase substrate solution $\left(0.05 \% \mathrm{DAB}, 0.015 \% \mathrm{H}_{2} \mathrm{O}_{2}, 0.01 \mathrm{M}\right.$ PBS buffer, $\left.\mathrm{pH} 7.2\right)$ to the sections for 5 min at room temperature. Negative control sections were incubated in the absence of the primary antibody or in the absence of the secondary antibody to verify the specificity of both antibodies in the DAB immunostaining procedure. ImageJ software, version 1.51a (NIH, USA; rsb.info.nih.gov/ij/) was used to analyze the $[\mathrm{MT}$ I+II] in DAB immunostained tissues and background subtraction was applied.

\section{Western blotting}

For this method, eight rats were examined for each diabetic and control group. Cell lysates were extracted in lysis buffer containing $250 \mathrm{mmol} / \mathrm{L} \mathrm{NaCl}, 50 \mathrm{mmol} / \mathrm{L} \mathrm{4-(2-hydroxyethyl)-1-}$ piperazineethanesulfonic acid (HEPES), $10 \%$ glycerol, $1 \%$ triton $\mathrm{X}-100,1.5 \mathrm{mmol} / \mathrm{L} \mathrm{MgCl} 2,1 \mathrm{mmol} / \mathrm{L}$ egtazic acid (EGTA), $10 \mathrm{mmol} / \mathrm{L} \mathrm{Na}_{4} \mathrm{P}_{2} \mathrm{O}_{7}, 1 \mathrm{mmol} / \mathrm{L} \mathrm{NaF}, 800 \mu \mathrm{mol} / \mathrm{L} \mathrm{Na}_{3} \mathrm{VO}_{4}, 7.5 \mathrm{pH}$, and centrifuged at $12000 \mathrm{~g}$ for $10 \mathrm{~min}$. Supernatant was collected, and protein was measured using Pierce bicinchoninic acid (BCA) Protein Assay Kit (Thermo Scientific, Mississauga, Ontario, Canada). Fifteen micrograms of lysates were separated by $15 \%$ sodium dodecyl sulfate polyacrylamide gel electrophoresis (SDS-PAGE) and transferred to a nitrocellulose membrane $(0.2 \mu \mathrm{m}, 162-0146$, Bio-Rad, Mississauga, Ontario, Canada) using a $10 \mathrm{mmol} / \mathrm{L}$ sodium tetraborate buffer. The membranes were blocked in $5 \%$ BSA in tris buffered saline-tween (TBS-T) buffer $(10 \mathrm{mmol} / \mathrm{L}$ Tris- $\mathrm{HCl}, \mathrm{pH} 7.5,150 \mathrm{mmol} / \mathrm{L} \mathrm{NaCl}$, $0.05 \%$ Tween 20 ) for $1 \mathrm{~h}$ at room temperature followed by overnight incubation at $4{ }^{\circ} \mathrm{C}$ with the metallothionein (1:2000, ab12228; Abcam) primary antibody. The blots were washed, incubated with horseradish peroxidase-conjugated secondary antibodies (anti-mouse, ab6728; Abcam), and visualized with a chemiluminescence system (Immun-Star Chemiluminescent; 1705070; Bio-Rad). The bands were analyzed using the Image J software, version 1.51a (NIH, USA; rsb.info.nih.gov/ij/).

\section{Detection of oxidized protein}

To detect changes in oxidative modification of proteins an Oxidized Protein Western Blot Kit (ab178020; Abcam) was used according to the manufacturer's specifications. Briefly the level of carbonyl groups that are introduced to the amino acid side chains after oxidative modification was determined. The kit derivatizes the carbonyl groups to a 2,4-dinitrophenyl-hydrazone (DNP) moiety. The DNP moiety can then be detected using anti-DNP antibodies. Five micrograms of protein per 
sample were derivatized as per the protocol given in the kit. These proteins where separated on a $10 \%$ SDS-PAGE gel and transferred onto a nitrocellulose membrane. After transfer and Ponceau S staining the membranes were blocked with $3 \%$ skim milk (in TBS with $0.1 \%$ Tween-20) for $1 \mathrm{~h}$ at room temperature. The nitrocellulose membrane was exposed to a primary anti-DNP antibody (1:1000) overnight at $4{ }^{\circ} \mathrm{C}$ and then to a secondary HRP-conjugated antibody (1:5000) for $1 \mathrm{~h}$ at room temperature. After every step, membranes were washed in TBS with $0.1 \%$ Tween-20. Protein bands were visualized with ECL (Immun-Star Chemiluminescent; 1705070; Bio-Rad). Band signal intensity was quantified by densitometry using the Image J software, version 1.51a (NIH, USA; rsb.info.nih. gov/ij/).

\section{Statistics}

All data values are presented as mean \pm SE. All statistical analyses were performed using the SPSS Statistics software, version 24 (SPSS Inc., Chicago, Illinois, USA) and $p<0.05$ was considered significant. Shapiro-Wilk tests revealed that all the data values were normally distributed. Bilateral Student's $t$ tests were performed to evaluate if there was a statistically significant difference in MT I+II protein expression between the different tissues.

\section{Results}

At 4 weeks after baseline, the STZ-induced rats exhibited an increase in blood glucose concentration and a decrease in body weight compared with the control rats (see Larsen et al. 2015, figure 2). Moreover, at the time of euthanasia, detectable levels of ketone bodies were only observed in STZinduced rats. Taken together, these observations confirmed the presence of diabetes in STZ-induced rats (Larsen et al. 2015).

For both methods (DAB immunostaining and Western blotting), all data values were equated to the MT I+II protein expression values in the control plantaris tissue to facilitate intra- and intergroup comparisons as a means to provide additional information.

\section{DAB immunostaining}

Immunostaining analysis revealed that the STZ-induced diabetic rats had a significantly higher $(p<0.05)$ MT protein expression than the control rats in the plantaris $(131.85 \pm 10.84$ vs. $100 \pm 6.23)$ and in the soleus muscle tissue (132.47 \pm 5.40 vs. $104 \pm 5.94)$ (Fig. 1). The difference in MT protein expression between the diabetic rats and the nondiabetic rats was slightly higher in the plantaris muscle compared with the soleus muscle (31.85\% vs. $28.28 \%$, no significant difference). The representative images of the DAB immunostainings are presented in Fig. 2.

\section{Western blotting}

To control for relative protein differences we used immunoblotting with a MT I+II specific antibody which values were normalized to the beta tubulin expression. We compared all data with the control values for plantaris tissue $(100 \pm 14.2)$ which indicated a significant $(p<0.05)$ increase in the MT expression in the diabetic plantaris tissue $(139 \pm 9.9)$. This method further indicated a significant upregulation between the control and diabetic soleus muscle $(120 \pm 7.4$ vs. $146 \pm 8.6, p<0.05$, respectively) (Fig. 3A). The representative Western blotting images are presented in Fig. 3B.

\section{Oxidative stress}

As a global measure of the effects of type 1 diabetes on oxidative stress we detected the amount of protein carbonyl formation. In both diabetic conditions there was a significant upregulation of the densitometry when compared with transferred total protein on the blotting membrane. The control was 


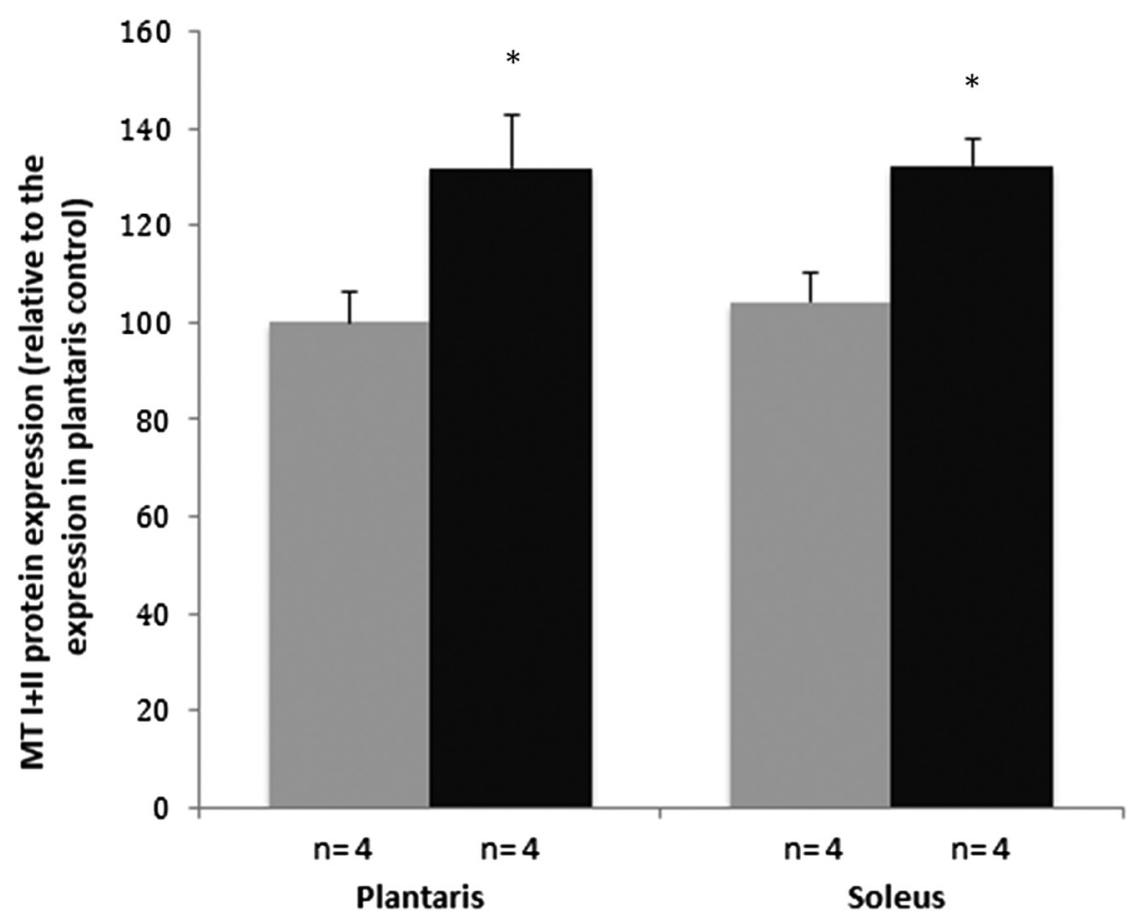

Fig. 1. Metallothionein (MT) protein expression in 3,3'-diaminobenzidine (DAB) immunostained plantaris and soleus muscle tissues. Black bars represent streptozotocin-induced diabetic animals and gray bars represent control animals. Data are mean \pm SE, control vs. diabetic. ${ }^{\star} p<0.05$.
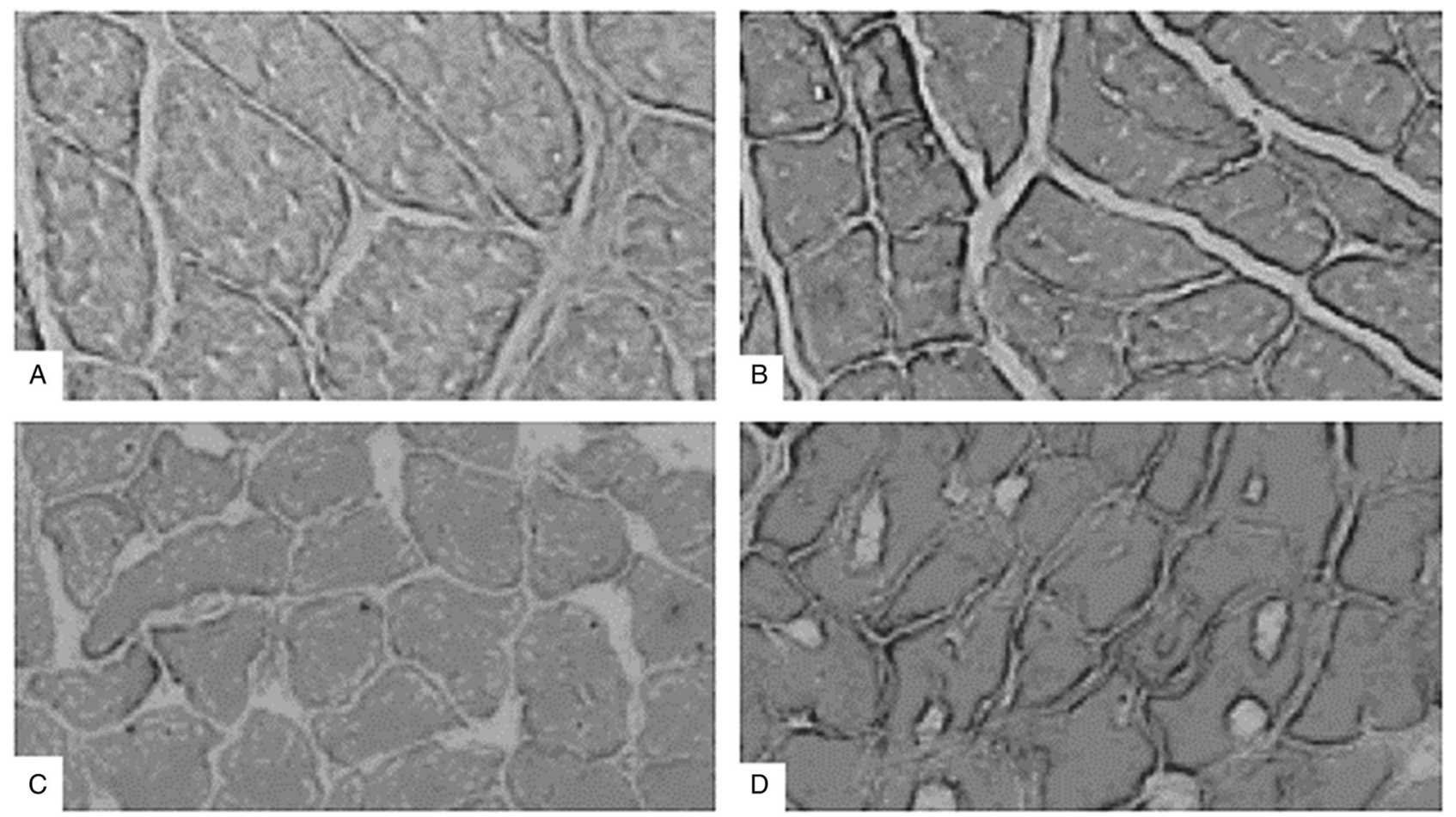

Fig. 2. Representative metallothionein (MT) I+II 3,3'-diaminobenzidine (DAB) immunostainings. (A) Control soleus muscle tissue, (B) diabetic soleus muscle tissue, (C) control plantaris muscle tissue, (D) diabetic plantaris muscle tissue. 


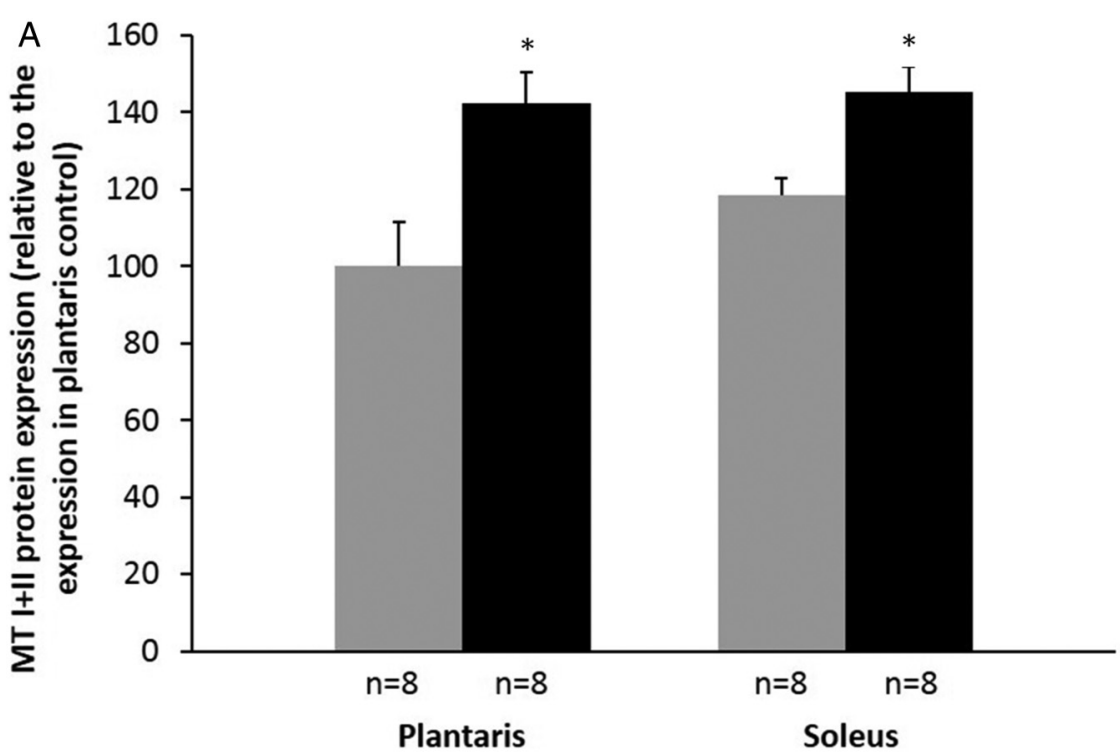

B

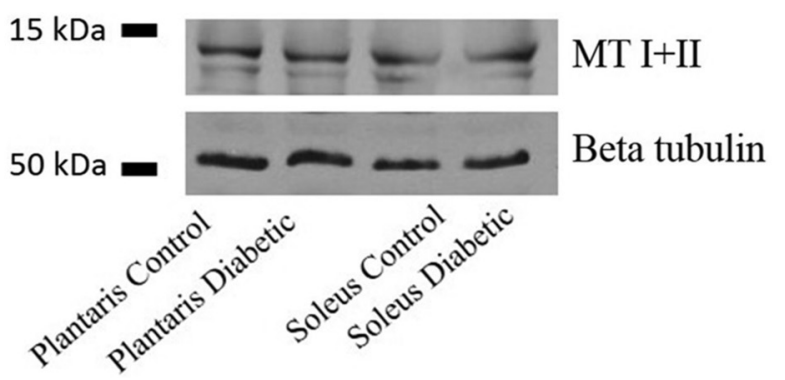

Fig. 3. (A) Metallothionein (MT) protein expression measured by Western blotting in plantaris and soleus muscle tissues. Black bars represent streptozotocin-induced diabetic animals and gray bars represent control animals. Data are mean \pm SE, control vs. diabetic. ${ }^{*} p<0.05$. (B) Representative MT I+II blot with beta tubulin as a loading control.

compared with diabetic plantaris tissue $(1 \pm 0.12$ vs. $1.42 \pm 0.08, p<0.05)$ as well as with diabetic soleus tissue ( $1 \pm 0.04$ vs. $1.76 \pm 0.10, p<0.05)$ (Fig。 4).

\section{Discussion}

The major finding of the present study is that MT protein expression increases in rat plantaris and soleus muscles in response to T1DM as demonstrated by DAB immunostaining and Western Blot analysis. Our results are in accordance with a previous study that reported increased MT I+II mRNA levels in the soleus and plantaris muscles of STZ-induced T1DM mice (Kivelä et al. 2006). Other studies involving DNA microarrays showed that STZ-induced diabetic rats exhibit a higher MT I expression in the gastrocnemius muscles (Lecker et al. 2004) and a higher MT I-II expression in the biceps femoris muscle (Willsky et al. 2006) compared with nondiabetic rats. The gastrocnemius muscle contains predominantly type 2D fibers (Cornachione et al. 2011), whereas the biceps femoris muscle is composed of similar proportions of type 1 and 2 fibers (Dahmane et al. 2006), which suggests that there is a consistent increase in MT protein expression in different muscle types associated with T1DM. Additionally, an increase in MT expression has been observed in other tissues including 


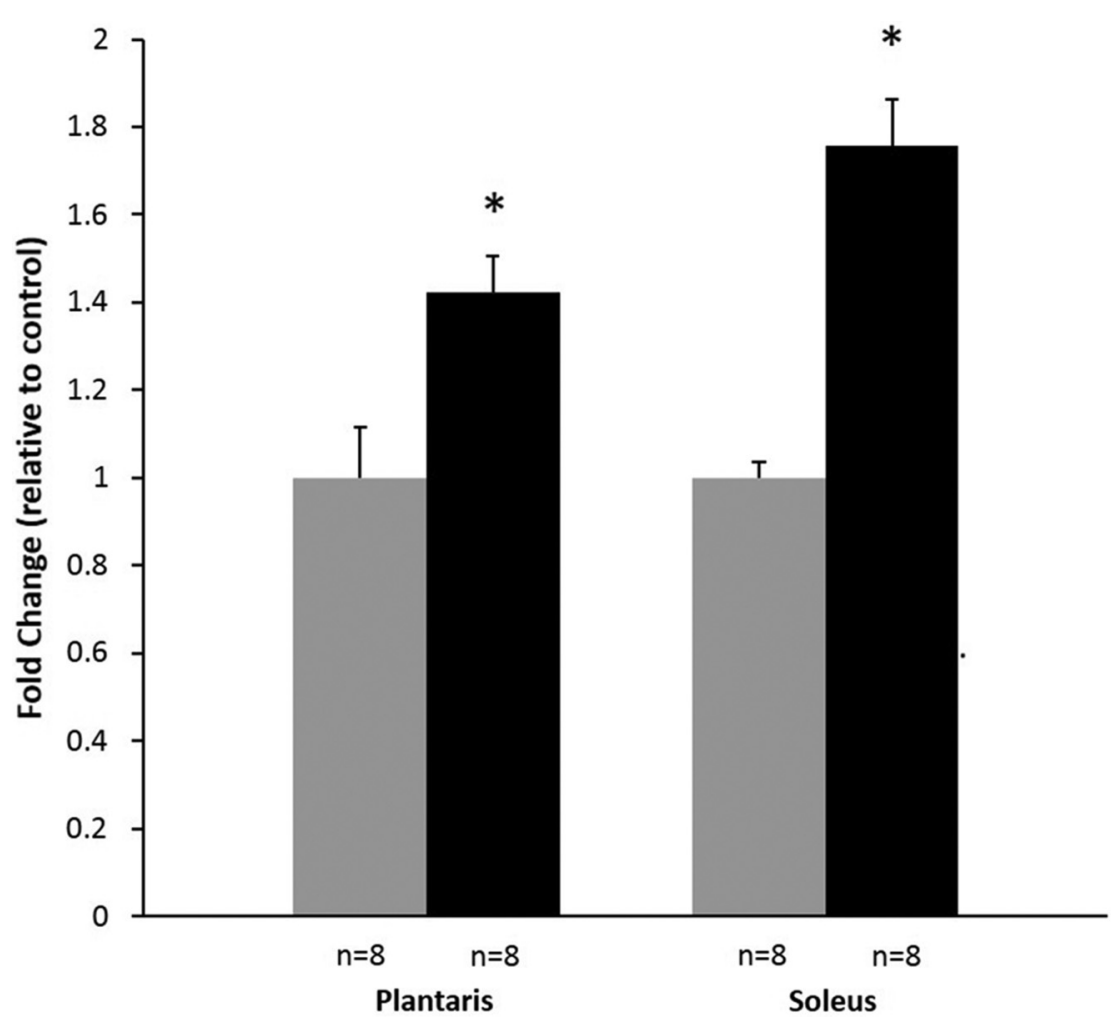

Fig. 4. Alterations in protein oxidation measured by Oxidized Protein Western Blot Kit following streptozotocininduced type 1 diabetes. The oxidative index (protein carbonyls) was substantially higher in the diabetic tissues when compared with the control. Black bars represent streptozotocin-induced diabetic animals and gray bars represent control animals. Data presented as mean $\pm \mathrm{SE}$, control vs. diabetic. ${ }^{\star} p<0.05$.

the liver and the kidneys (Cai et al. 2002) and in the cardiac tissue of STZ-induced T1DM mice (Song et al. 2005).

Skeletal muscle is the largest insulin-sensitive organ in the body and has consequently a central role in glucose homeostasis (Coleman et al. 2015). In addition, the skeletal muscle has a primordial role in movement and is one of the most important tissues in energy utilization. Moreover, therapies focused on skeletal muscle could contribute to delay diabetes-related complications that affect other organs (Coleman et al. 2015).

It is well established that the switch from glucose to fatty acid utilization as an energy source in T1DM results in up-regulation of fatty acid $\beta$ oxidation enzymes (Yechoor et al. 2002), which consequently leads to increased ROS production. In the present study, protein oxidation was found to be elevated for diabetic plantaris and soleus muscles when compared with the control condition, which confirmed the presence of oxidative stress in the diabetic tissue. The observed increase in [MT I+II] as a response to T1DM in skeletal muscle suggests an increased antioxidant response to this oxidative stress. Different mechanisms have been proposed for MT's protection against diabetesinduced oxidative stress (Zatta 2008). In the heart, MT may be involved in the suppression of the mitochondrial cytochrome $\mathrm{c}$ release-dependent apoptotic pathway and in the attenuation of the calcium handling abnormalities that are associated with diabetes cardiac complications (Zatta 2008). Similar mechanisms may be responsible for MT's protective effect against oxidative stress in the skeletal muscle. 
Previous studies have reported that the overexpression of MT in T1DM animal models leads to the prevention of diabetic cardiomyopathy (Liang et al. 2002) and nephrotoxicity (Jin et al. 1996). Furthermore, the disease complications in T2DM have been associated with a decrease in antioxidant capacity (Lodovici et al. 2008). Because both T2DM and T1DM are chronic diseases that involve oxidative stress, the complications of T1DM could be associated to similar phenomena. This suggests that at the onset of T1DM the MT expression increases in response to the oxidative stress, but as complications develop the protective effects of MT decrease. Considering that antioxidant supplementation studies are being performed in humans and that zinc-induced MT therapy has already been applied in humans suffering from Wilson's disease with satisfactory results (Sturniolo et al. 1999), MT overexpression could be a potential therapy to prevent T1DM complications.

A slightly larger difference in MT expression between the diabetic rats and the control rats was observed in the plantaris muscle than in the soleus muscle ( $31.85 \%$ vs. $28.28 \%$, not statistically significant), which represents a trend that could be of interest considering the different fiber composition of these muscle tissues. One reason that could explain this observation is advanced glycation end products, which are formed as a consequence of oxidative stress and have been shown to cause a greater negative effect on fast-twitch fibers than in slow-twitch fibers (Krause et al. 2011). Another reason that could explain the increased difference in $[\mathrm{MT}]$ observed in the plantaris muscle compared with the soleus muscle is the difference in the mitochondrial content in both muscle types. Due to their strong oxidative capacity, mitochondria are the main target of oxidative stress damage in diabetes mellitus (Asmat et al. 2016). Slow-twitch muscle fibers (e.g., soleus) have a higher mitochondrial content than fast-twitch muscle fibers (e.g., plantaris) (Sanchez et al. 2014). This raises the possibility that for the same amount of accumulated fatty acids, the mitochondria of the plantaris would be affected faster, whereas the effect would be delayed in the soleus. As a result, an augmented relative amount of mitochondria would be damaged and destroyed in the plantaris muscle, which would result in a higher and faster increase in ROS production compared with the soleus muscle. Both of these possibilities suggest that fast-twitch fibers exhibit higher levels of oxidative stress than slow-twitch fibers, which could be associated with a subsequent higher increase in MT expression in the plantaris muscle compared with the soleus muscle.

In conclusion, the present study supports the hypothesis that MT I+II is implicated in the response of the soleus and plantaris muscles to T1DM. These results are consistent with previous studies performed in skeletal muscle and other organs of STZ-induced T1DM rodents. Although hyperglycemia and the subsequent increase in ROS have been studied in detail, relatively little is known about how the antioxidant MT is affected. We report that the MT I+II protein expression increases in both the plantaris and soleus muscles of STZ-induced diabetic rats. Subsequent studies on this topic should focus on the downstream pathways involved, including changes at the mitochondria level. A more in-depth understanding of the molecular mechanisms that allow MT's protection against the oxidative stress observed in diabetes could lead to a novel therapeutic strategy for this disease and its associated complications.

\section{Acknowledgements}

Pamela Mondragon's work was supported by an NSERC Undergraduate Summer Research Award (USRA).

\section{Author contributions}

$\mathrm{AB}$ conceived and designed the study. $\mathrm{PM}$ performed the experiments/collected the data. $\mathrm{PM}$ and $\mathrm{AB}$ analyzed and interpreted the data. $\mathrm{PM}$ and $\mathrm{AB}$ contributed resources. $\mathrm{PM}$ and $\mathrm{AB}$ drafted or revised the manuscript. 


\section{Competing interests}

The authors have declared that no competing interests exist.

\section{Data accessibility statement}

All relevant data are within the paper.

\section{References}

Asmat U, Abad K, and Ismail K. 2016. Diabetes mellitus and oxidative stress-a concise review. Saudi Pharmaceutical Journal, 24(5): 547-553. DOI: 10.1016/j.jsps.2015.03.013

Cai L. 2006. Suppression of nitrative damage by metallothionein in diabetic heart contributes to the prevention of cardiomyopathy. Free Radical Biology and Medicine, 41(6): 851-861. PMID: 16934665 DOI: 10.1016/j.freeradbiomed.2006.06.007

Cai L, Chen S, Evans T, Cherian MG, and Chakrabarti S. 2002. Endothelin-1-mediated alteration of metallothionein and trace metals in the liver and kidneys of chronically diabetic rats. International Journal of Experimental Diabetes Research, 3(3): 193-198. DOI: 10.1080/15604280214281

Carpenè E, Andreani G, and Isani G. 2007. Metallothionein functions and structural characteristics. Journal of Trace Elements in Medicine and Biology, 21: 35-39. PMID: 18039494 DOI: 10.1016/j. jtemb.2007.09.011

Coleman SK, Rebalka IA, D’souza DM, and Hawke TJ. 2015. Skeletal muscle as a therapeutic target for delaying type 1 diabetic complications. World Journal of Diabetes, 6(17): 1323-1336. PMID: 26674848 DOI: 10.4239/wjd.v6.i17.1323

Cornachione AS, Benedini-Elias PCO, Polizello JC, Carvalho LC, and Mattiello-Sverzut AC. 2011. Characterization of fiber types in different muscles of the hindlimb in female weanling and adult Wistar rats. Acta Histochemica et Cytochemica, 44(2): 43-50. PMID: 21614165 DOI: 10.1267/ahc.10031

Dahmane R, Djordjevič S, and Smerdu V. 2006. Adaptive potential of human biceps femoris muscle demonstrated by histochemical, immunohistochemical and mechanomyographical methods. Medical and Biological Engineering and Computing, 44(11): 999-1006. PMID: 17024467 DOI: 10. 1007/s11517-006-0114-5

Giacco F, and Brownlee M. 2010. Oxidative stress and diabetic complications. Circulation Research, 107(9): 1058-1070. PMID: 21030723 DOI: 10.1161/CIRCRESAHA.110.223545

Jin T, Nordberg G, Sehlin J, and Vesterberg O. 1996. Protection against cadmium-metallothionein nephrotoxicity in streptozotocin-induced diabetic rats: role of increased metallothionein synthesis induced by streptozotocin. Toxicology, 106(1): 55-63. DOI: 10.1016/0300-483X(95)03164-B

King GL, and Loeken MR. 2004. Hyperglycemia-induced oxidative stress in diabetic complications. Histochemistry and Cell Biology, 122(4): 333-338. PMID: 15257460 DOI: $10.1007 /$ s00418004-0678-9

Kivelä R, Silvennoinen M, Touvra A-M, Lehti TM, Kainulainen H, and Vihko V. 2006. Effects of experimental type 1 diabetes and exercise training on angiogenic gene expression and capillarization in skeletal muscle. The FASEB Journal, 20(9): 1570-1572. PMID: 16816123 DOI: 10.1096/fj.05-4780fje 
Krause MP, Riddell MC, and Hawke TJ. 2011. Effects of type 1 diabetes mellitus on skeletal muscle: clinical observations and physiological mechanisms. Pediatric Diabetes, 12(4pt1): 345-364. PMID: 20860561 DOI: 10.1111/j.1399-5448.2010.00699.x

Larsen S, Scheede-Bergdahl C, Whitesell T, Boushel R, and Bergdahl A. 2015. Increased intrinsic mitochondrial respiratory capacity in skeletal muscle from rats with streptozotocin-induced hyperglycemia. Physiological Reports, 3(7): e12467. PMID: 26197936 DOI: 10.14814/phy2.12467

Lecker SH, Jagoe RT, Gilbert A, Gomes M, Baracos V, Bailey J, et al. 2004. Multiple types of skeletal muscle atrophy involve a common program of changes in gene expression. The FASEB Journal, 18(1): 39-51. PMID: 14718385 DOI: 10.1096/fj.03-0610com

Liang Q, Carlson EC, Donthi RV, Kralik PM, Shen X, and Epstein PN. 2002. Overexpression of metallothionein reduces diabetic cardiomyopathy. Diabetes, 51(1): 174-181. PMID: 11756338 DOI: 10. 2337/diabetes.51.1.174

Liang T, Zhang Q, Sun W, Xin Y, Zhang Z, Tan Y, et al. 2015. Zinc treatment prevents type 1 diabetesinduced hepatic oxidative damage, endoplasmic reticulum stress, and cell death, and even prevents possible steatohepatitis in the OVE26 mouse model: important role of metallothionein. Toxicology Letters, 233(2): 114-124. PMID: 25617602 DOI: 10.1016/j.toxlet.2015.01.010

Lodovici M, Giovannelli L, Pitozzi V, Bigagli E, Bardini G, and Rotella CM. 2008. Oxidative DNA damage and plasma antioxidant capacity in type 2 diabetic patients with good and poor glycaemic control. Mutation Research/Fundamental and Molecular Mechanisms of Mutagenesis, 638(1): 98-102. DOI: 10.1016/j.mrfmmm.2007.09.002

Liu C, Mathews CE, and Chen J. 2016. Oxidative stress and type 1 diabetes. In Oxidative stress and antioxidant protection: the science of free radical biology and disease. Edited by D Armstrong and RD Stratton. John Wiley \& Sons, Inc, Hoboken, New Jersey. DOI: 10.1002/9781118832431.ch19

Maritim AC, Sanders RA, and Watkins JB III. 2003. Diabetes, oxidative stress, and antioxidants: a review. Journal of Biochemical and Molecular Toxicology, 17(1): 24-38. PMID: 12616644 DOI: 10. 1002/jbt.10058

Powers SK, Ji LL, Kavazis AN, and Jackson MJ. 2011. Reactive oxygen species: impact on skeletal muscle. Comprehensive Physiology, 1(2): 941-969. PMID: 23737208 DOI: 10.1002/cphy.c100054

Sanchez B, Li J, Bragos R, and Rutkove SB. 2014. Differentiation of the intracellular structure of slowversus fast-twitch muscle fibers through evaluation of the dielectric properties of tissue. Physics in Medicine \& Biology, 59(10): 2369-2380. PMID: 24743385 DOI: 10.1088/0031-9155/59/10/2369

Song Y, Wang J, Li Y, Du Y, Arteel GE, Saari JT, et al. 2005. Cardiac metallothionein synthesis in streptozotocin-induced diabetic mice, and its protection against diabetes-induced cardiac injury. The American Journal of Pathology, 167(1): 17-26. PMID: 15972948 DOI: 10.1016/S0002-9440(10) $62949-5$

Sturniolo GC, Mestriner C, Irato P, Albergoni V, Longo G, and D'Incà R. 1999. Zinc therapy increases duodenal concentrations of metallothionein and iron in Wilson's disease patients. The American Journal of Gastroenterology, 94(2): 334-338. PMID: 10022625 DOI: 10.1111/j.1572-0241.1999.851_w.X

Van Belle TL, Coppieters KT, and Von Herrath MG. 2011. Type 1 diabetes: etiology, immunology, and therapeutic strategies. Physiological Reviews, 91(1): 79-118. PMID: 21248163 DOI: 10.1152/ physrev.00003.2010 
Willsky GR, Chi L-H, Liang Y, Gaile DP, Hu Z, and Crans DC. 2006. Diabetes-altered gene expression in rat skeletal muscle corrected by oral administration of vanadyl sulfate. Physiological Genomics, 26(3): 192-201. PMID: 16684804 DOI: 10.1152/physiolgenomics.00196.2005

World Health Organization. 2016. Global report on diabetes. World Health Organization, Geneva, Switzerland.

Wu J and Yan LJ. 2015. Streptozotocin-induced type 1 diabetes in rodents as a model for studying mitochondrial mechanisms of diabetic $\beta$ cell glucotoxicity. Diabetes, Metabolic Syndrome and Obesity: Targets and Therapy, 8: 181-188. DOI: 10.2147/DMSO.S82272

Yang J, and Cherian MG. 1994. Protective effects of metallothionein on streptozotocin-induced diabetes in rats. Life Sciences, 55(1): 43-51. DOI: 10.1016/0024-3205(94)90080-9

Yechoor VK, Patti M-E, Saccone R, and Kahn CR. 2002. Coordinated patterns of gene expression for substrate and energy metabolism in skeletal muscle of diabetic mice. Proceedings of the National Academy of Sciences of the United States of America, 99(16): 10587-10592. PMID: 12149437 DOI: 10. 1073/pnas.142301999

Zatta P. 2008. Metallothioneins in biochemistry and pathology. World Scientific Publishing Co Pte Ltd, Singapore. 336 p. 